\title{
Depression in physical illness
}

\author{
R Smyth \\ Consultant Psychiatrist, Department of Psychological Medicine, Royal Infirmary of Edinburgh, Edinburgh
}

ABSTRACT Depressive illnesses are common in the general population and are significantly more common in those with physical illnesses. The symptoms of depression may go unrecognised in medical patients, with consequent underdiagnosis and under-treatment. Co-morbid depression is associated with increased morbidity, poorer function, increased healthcare costs and increased mortality. Depressive illnesses are amenable to treatment by both pharmacological and psychological means. Successful treatment can result in improved quality of life as well as improved function, mortality and overall outcome in the physical disorder. It is therefore important that medical doctors are aware of the symptoms, diagnosis and management of depressive illness.
Published online September 2009

Correspondence to R Smyth, Department of Psychological Medicine, Royal Infirmary of Edinburgh, Edinburgh EHI6 4SA, UK

tel. $+44(0) 1312421398$

e-mail

roger.smyth@luht.scot.nhs.uk

KEYWORDS Depressive illness, liaison psychiatry, mood disorder

DECLARATION OF INTERESTS No conflict of interests declared.

Depressive illness is a disorder of mood where there is sustained pervasive low mood and anhedonia (loss of the ability to experience pleasure). These core symptoms are often associated with biological depressive features such as disturbed sleep, loss of appetite, impaired concentration and memory and lack of libido. The thoughts of depressed patients can come to be dominated by depressive cognitions - persistently gloomy thoughts and a negative appraisal of both the current situation and the future. At their most extreme these thoughts can give rise to hopelessness and thoughts or plans for suicide. Depressive illnesses can be of very variable severity, from mild and transient to severe and prolonged. Short-lived depressive symptoms are within the range of normal experience; they are of diagnostic significance if they are present for more than two weeks and of sufficient severity to interfere with normal activities.

\section{DEPRESSION IN THE PHYSICALLY ILL}

Depressive illnesses are more common in patients with physical illness than those without. Prevalence rates for depression are $\sim 5 \%$ in primary care patients, $\sim 10 \%$ in medical outpatients and 10-20\% in medical inpatients (with some studies reporting rates as high as $30 \%$ ). Rates of depression tend to increase with the severity of the medical illness: for example, rates are high in patients in the aftermath of myocardial infarction, but similar to general population levels for those with mild angina or hypertension.

Physically ill patients with co-morbid depression have increased morbidity related to the depressive illness itself as well as occasional mortality via suicide. Additionally these patients have morbidity related to decreased compliance with medical treatment, reduced
TABLE I Risk factors for the development of depression in the physically ill

\begin{tabular}{|l|}
\hline Personal or family history of depression \\
\hline Severe or life-threatening illness \\
\hline Chronic, debilitating, disfiguring or painful illness \\
\hline Unpleasant and demanding treatment \\
\hline $\begin{array}{l}\text { Lack of family or social support; other adverse social } \\
\text { circumstances }\end{array}$ \\
\hline Co-morbid alcohol or drug misuse \\
\hline Drug treatments that cause depression as a side effect \\
\hline $\begin{array}{l}\text { Illnesses or treatments affecting the neurological or } \\
\text { endocrine systems }\end{array}$ \\
\hline
\end{tabular}

overall quality of life, reduced functional levels and increased overall mortality compared with nondepressed controls. They are also found to have longer hospital stays and increased healthcare costs.

Identification and treatment of depressive illness is more difficult in those with physical illness (see Table I), but successful treatment offers the possibility of decreased psychological morbidity, reduced healthcare costs, improved compliance and participation in rehabilitation, and reduced overall mortality.

The co-occurrence of depression and physical illness can arise in a number of ways. Firstly, the physical illness can cause or exacerbate the depression; secondly, the depression can cause or exacerbate the physical illness; and, thirdly, the two can have a common cause. Additionally, any co-occurrence may be coincidental as depressive illnesses are common and can be expected to co-occur with other common physical illnesses from time to time. 


\section{Depression caused or exacerbated by physical illness}

Physical illness can cause depression either by a (presumed) direct biological effect, via psychological factors related to the physical disease or via the depressant effects of the treatment or treatment side effects.

- Biological effect A presumed direct biological effect may arise via the endocrine system (e.g.hypothyroidism, Cushing's disease, parathyroid disease, Addison's disease) or the neurological system (e.g. post stroke, Parkinson's disease, multiple sclerosis, Huntington's disease, post head injury) or via indirect mechanisms in cardiac disease (e.g. post myocardial infarction, heart failure), infectious diseases (e.g. human immunodeficiency virus [HIV] associated opportunistic infections, hepatitis $\mathrm{C}$, infectious mononucleosis) or systematic disease (e.g. systemic lupus erythematosus [SLE], rheumatoid arthritis, cancer).

- Psychological cause Depression is often psychologically related to events that symbolise loss or change, events that are common in the context of physical ill health (e.g. lethal or life-threatening illness, amputation, loss of sexual function or child-bearing ability, loss of ability to work, change in appearance or body image).

- Treatment side effects Depression can be a predictable or idiosyncratic side effect of multiple drugs (e.g. corticosteroids, L-dopa, beta blockers, cytotoxics, digoxin, calcium channel blockers, aminophylline, nonsteroidal anti-inflammatories [NSAIDs], cimetidine, metoclo-pramide, alpha-interferon, anticonvulsants, isotretinoin).

Physical illness caused or exacerbated by depression Physical illness can be directly attributable to a depressive illness, e.g. fulminant hepatic failure following paracetamol overdose taken during a depressive relapse. More usually, however, mood disorder worsens physical ill health more indirectly, by interfering with compliance and participation in rehabilitation and by associated poor diet, lack of exercise and overall poorer self-care.

\section{Depression and physical illness with a common cause}

Occasionally physical illness and the depressive illness can be seen to share a common cause; e.g. a sequence of stressful life events acting as precipitants to both a myocardial infarction and an episode of depression.

\section{THE SYMPTOMS OF DEPRESSION}

Depressive illnesses can present in a multitude of ways, including apparent low mood or tearfulness, expressions of hopelessness about recovery, lack of interest in rehabilitation, apparent personality change, irritability or aggression. There may be an increase in somatic complaints despite no apparent worsening of the underlying physical condition or apparent cognitive impairment (pseudo-dementia). Nursing staff are often
TABLE 2 Diagnostic and Statistical Manual IV (DSM-IV) criteria for a major depressive episode Depressed mood most days, as indicated by either
subjective report or observation made by others*

Markedly diminished interest or pleasure in all, or almost all, activities most days*

Significant weight loss when not dieting or weight gain (e.g. a change of more than $5 \%$ of body weight in a month), or decrease or increase in appetite nearly every day

Psychomotor agitation or retardation nearly every day

Fatigue or loss of energy nearly every day

Feelings of worthlessness or excessive or inappropriate guilt nearly every day (not merely self-reproach or guilt about being sick)

Diminished ability to think or concentrate, or indecisiveness, nearly every day

Recurrent thoughts of death (not just fear of dying),

recurrent suicidal ideas without a specific plan, or a suicide attempt or a specific plan for committing suicide

At least five of the above symptoms, and at least one of the symptoms marked $(*)$ must have been present for the previous two weeks for a DSM-IV diagnosis of major depressive episodes. Symptoms can be identified by subjective report or objective observation.

more alert to such symptoms than medical staff, who may see the patient only briefly and who will inevitably focus on physical symptoms.

Depressive illness is a recognised clinical syndrome rather than a specific diagnosis, and currently the exact aetiology and pathology are unknown. There is hence no definitive diagnostic test available. The diagnosis of depression is made on the basis of operational diagnostic criteria. The two best known and most frequently used are the International Classification of Diseases (ICD-I0), published by the World Health Organization and used around the world, and the Diagnostic and Statistical Manual (DSM-IV), published by the American Psychiatric Association and used in the USA and for research purposes (see Table 2).

However diagnosed, all depressive disorders are characterised by a lowering of mood which is severe and persistent. Further diagnostic symptoms can be divided into three main areas: diminished mood/enjoyment, biological depressive features and depressive cognitions.

\section{DIFFERENTIAL DIAGNOSIS OF DEPRESSION IN PHYSICAL ILLNESS}

Because they are broad clinically described syndromes rather than specific disorders, the differential diagnosis for mental disorders is usually short. In patients with physical illness the main distinction to be made is between the physical illness alone (with some of its manifestations, e.g. insomnia or bradykinesia, presenting as apparent depression) and true co-morbid depressive illness. 
The biological depressive features of insomnia, loss of appetite, poorer concentration and memory, and lack of libido are much less diagnostically useful in patients with physical illness due to the possibility of diagnostic confusion with symptoms of the physical disorder itself. Subjectively described and objectively observed low mood (particularly if pervasive and with characteristic diurnal variation) and depressive cognitions are of much more diagnostic value.

Conditions to be considered in the differential diagnosis include:

- Understandable emotional reactions to physical ill health;

- Adjustment reactions - short-lived episode of depressive symptoms with a clear relationship to recent stressful events;

- Delirium. Patients with hypoactive delirium may present as inactive, unco-operative and 'depressed', and so basic cognitive testing (e.g. abbreviated mental test [AMT], mini mental state examination [MMSE]) is an important part of the assessment of patients at risk;

- Drug or alcohol misuse or dependence may present with physical symptoms but can also be associated with mood disturbance;

- In true organic depression (e.g. related to hypothyroidism) one should treat the underlying physical condition first and then reassess the mood before considering specific treatment of depression.

\section{ASSESSMENT OF PATIENTS WITH APPARENT DEPRESSIVE SYMPTOMS}

Clinicians should have a low index of suspicion for the diagnosis of depression in the physically ill. A major determinant as to whether depressive illness will be diagnosed is the willingness of the medical team to ask about symptoms and the whole treating team to be alert to the possibility (see Table 3).

When assessing patients with apparent low mood, care should be taken to ensure the right environment for the assessment. There should be sufficient privacy to allow discussion of sensitive topics and sufficient time available to allow for full discussion without 'feeling rushed'. Often the interview will break from a formal structure in order to follow up information as it becomes available. In general, however, one should try to cover the following areas:

- Are there signs of cognitive impairment? Particularly in inpatients, an apathetic, 'depressed' presentation may represent hypoactive delirium. Consider performing a basic test of cognitive function (e.g. AMT, MMSE).

- How does the patient himself describe his mood? Depression is a cognitive process and its symptoms are subjective experiences. Sometimes patients 'look depressed' but are not; the reverse, of course, can
TABLE 3 Reasons why depression may be under-diagnosed in physically ill patients

Lack of awareness of symptoms of depression and breadth of possible presentations

Focus on physical symptoms during history taking

Lack of time or a suitable environment during medical consultation to explore mental health symptoms

Similarity between symptoms of depression and physical illness

Making assumptions that depressed mood after physical illness is an understandable reaction to ill health ('l'd be depressed if I was in his position') and hence not amenable to treatment

Unwillingness of the patient (or doctor) to consider or discuss mental health problems

Presupposition that antidepressants will be contraindicated given the physical health problem

TABLE 4 Suggested questions to explore symptoms of low mood

\begin{tabular}{|l|}
\hline How have you been feeling recently? \\
\hline Have you been low in spirits? \\
\hline Have you been able to enjoy the things you usually enjoy? \\
\hline How has your appetite been lately? \\
\hline How has your sleep been? \\
\hline Have you had your usual level of energy? \\
\hline $\begin{array}{l}\text { Have you been able to concentrate on newspaper articles } \\
\text { or your favourite television or radio programmes? }\end{array}$ \\
\hline
\end{tabular}

also be true. Ask about diurnal variation in mood. In depressed patients the mood is often lowest in the morning, with improvement later in the day.

- Consider the core and other symptoms of depression described above. The biological depressive features are often less useful in patients with physical illness as they may be mimicked or masked by the symptoms of the physical illness. Nonetheless they should be asked about (see Table 4). Is there impairment of sleep, appetite, energy levels, concentration and libido?

- The most important features diagnostically are the cognitive features, particularly patients' view of the future: are they hopeless about the prospects for physical recovery? Have they lost the ability to enjoy formerly pleasurable activities (anhedonia)? Their activities may, of course, be limited by physical ill health, but, for example, are they still able to enjoy family visits?

- If possible try to talk to family members or others who know the patient well, who may be able to comment on the patient's current mood, previous treatment and severity.

- Screening tools such as the Hospital Anxiety and Depression Scale (HADS), the Beck Depression Inventory (BDI) and the Geriatric Depression Scale (GDS) can be useful in assessing the severity of disorder and measuring improvement, but do not replace clinical assessment in diagnosis. 


\section{TREATMENT}

Effective treatment for depression offers the possibility of improvement in mood (which is, of course, valuable in itself) but also the possibility of improvements in compliance, participation in rehabilitation, decreased length of hospital stay and an overall reduction in morbidity, mortality and eventual disability.

The treatment of depression in the physically ill uses the same methods and principles as in the physically well. The treating doctor should consider the use of pharmacological, psychological and social treatments, guided by symptomatology, history and patient preference. Treatments for co-morbid depressive illness can be broadly considered under the following headings:

- Practical interventions. For example, education about the physical disorder (to counteract unrealistic worries), improved socialisation (e.g. move from side room, facilitate visits out), optimisation of pain and symptom control.

- Psychological support. Support for the patient's emotional and psychological needs is a key nursing role and should not be neglected alongside the technical aspects of care. Other allied health professionals will also play a role, with psychological support alongside practical rehabilitation being especially valuable.

- Specific psychological treatments. While formal psychological therapies are often unavailable in general hospitals, aspects of, for example, cognitive behavioural therapy are often incorporated into physical rehabilitation and pain management programmes.

- Consideration of drug treatment. Antidepressant drugs are effective in those with physical illness, although there is often need for caution in drug choice and dosing schedule. It is not unreasonable to consider a therapeutic trial of antidepressant medication in cases of diagnostic doubt.

\section{Psychological treatments}

Psychological treatments (or 'talking therapies') are effective and widely used to treat mood disorders. There are a range of different psychological therapies each with its own rationale and method: examples include cognitive behavioural therapy (CBT), interpersonal therapy (IPT), dynamic (e.g. Freudian) therapy and group therapies. A major determinant in therapy choice will often be local availability and waiting times.

Psychological treatments have some advantages in treating depression in the physically ill in that they avoid the risk of drug interactions or drug side effects. Unfortunately, there are often significant practical difficulties in organising regular therapy attendance (e.g. for an hour per week for many weeks) for patients with physical health problems. Additionally such therapies often have long waiting times, or are simply unavailable in many areas.

\section{Drug treatment}

Antidepressant drug treatments are effective in the treatment and prophylaxis of depressive illness. These drugs are usually well tolerated, even in patients with physical illnesses, and do not induce physical dependence. Antidepressant drug treatment should be considered in patients with persisting depressive symptoms that are interfering with their quality of life or overall function.

Antidepressant drugs fall into two broad classes by pharmacological action: those that increase the amount of intrasynaptic neurotransmitter by blocking presynaptic reuptake, such as selective serotonin reuptake inhibitors (SSRIs) and tricyclic antidepressants (TCAs), and those that increase the amount of intrasynaptic neurotransmitter by blocking its breakdown, the monoamine oxidase inhibitors (MAOls). Clinically, however, one tends to consider four groupings: SSRIs (e.g. fluoxetine, citalopram), TCAs (e.g. imipramine, amitriptyline), MAOls (e.g. phenelzine, moclobemide) and other, novel drugs (e.g. venlafaxine, mirtazapine).

In current clinical practice SSRIs are generally first-line drugs, with newer antidepressants used as second-line drugs for SSRI non-responders, and TCAs and MAOls used in treatment-resistant cases. Where a patient has a history of a previous good response to an antidepressant, that drug is usually considered the first choice drug for them in future episodes of depression. Antidepressants have a slow onset of action and clinical response cannot be expected before |4-2| days after starting the drug.

For patients with physical illness, particularly if there is liver or kidney impairment, drugs may have to be introduced at a lower starting dose and increased in dose more cautiously. The British National Formulary and the Psychotropic Drug Directory are invaluable sources of guidance for decisions regarding psychotropic prescribing.

\section{Drug treatment in specific disorders}

- Heart disease There is a significant increase in the incidence of depression in patients following myocardial infarction (MI) which is associated with an increased mortality. Antidepressants are best avoided in the first month after Ml; where necessary SSRIs are the first-line drugs. Selective serotonin reuptake inhibitors are also first-line in patients at risk of arrhythmias.

- Liver disease All antidepressants are cleared by the liver so there is a risk of accumulation and potentiation of side effects in those with liver failure, regardless of cause. Selective serotonin reuptake inhibitors at halfnormal starting doses with more cautious dose increases are the drugs of choice, with citalopram usually well-tolerated in liver disease. Tricyclic anti- 
depressants can precipitate hepatic encephalopathy and should be used with caution. Monoamine oxidase inhibitors should be avoided.

- Kidney disease Again there is a risk of drug accumulation and potentiation of side effects. Lower starting doses, cautious dose increases and consideration of divided dosing can avoid this risk. Tricyclic antidepressants may be the drugs of choice, avoiding lofepramine.

- Diabetes Depression is common in patients with both type I and type 2 diabetes, with rates as high as $30 \%$ seen in those with end-organ complications. While SSRIs are the first-line antidepressants, TCAs are often used at low dosage to treat neuropathy. Fluoxetine carries increased potential for hypoglycaemia and should be avoided, as should mirtazapine, which can cause undesirable weight gain.

\section{KEY POINTS}

- Depressive illness is a common disorder which occurs much more frequently in those with physical illness than in the population at large.

- It is diagnosed using operational diagnostic criteria such as pervasive low mood and loss of the ability to experience pleasure, coupled with biological features of depression and depressive cognitions.

- Co-morbid depression is associated with poorer outcomes in physical illness, with increased morbidity, decreased functional level, increased healthcare costs and overall increased mortality.

- Depression is under-recognised in medical patients but, with increased awareness and some modification of diagnostic methods, can be successfully identified.

- Psychological and drug treatments are available that are effective in depression. Successful treatment is associated with improved quality of life and improved outcomes for the physical disease.

\section{FURTHER READING}

- Bazire S. The psychotropic drug directory 2009. Aberdeen: HealthComm UK; 2008.

- National Institute for Health and Clinical Excellence (NICE). Management of depression in primary and secondary care. London: NICE; 2007. Available from: http://guidance.nice.org.uk/CG23

- Peveler R, Carson A, Rodin G. ABC of psychological medicine: depression in medical patients. BMJ 2002; 325:149-52. doi: I0.1/361 bmj.325.7356.149

\section{SELF-ASSESSMENT QUESTIONS}

I. An 83-year-old woman is admitted to an orthopaedic ward with a fractured neck of femur. She undergoes operative repair after which her recovery is complicated by a wound infection. She is not noted to be depressed prior to the operation. She has no history of mood disorder or cognitive impairment. Six days post-operatively she presents to nursing and therapy staff as flat, apathetic and uninterested in rehabilitation. Which is the correct immediate action?

A. Trial of antidepressant.

B. Trial of antipsychotic.

C. Cognitive assessment.

D. Psychology review.

E. Transfer to specialist rehabilitation unit.

2. A 62-year-old man is admitted for chemotherapy for lymphoma. His wife reports that he has appeared depressed in recent weeks. He is very reluctant to discuss mood symptoms and appears to be trying to 'put on a brave face' in the interview situation. Which of the following features of depressive illness is the most useful diagnostically in this situation?

A. Loss of appetite.

B. Lack of interest in pleasurable activities (e.g. seeing his family).

C. Weight loss.

D. Sleep disturbance.

E. Poor concentration.

3. A 42-year-old woman with a diagnosis of end-stage renal disease is being seen on the dialysis unit. She has a history of recurrent depressive illness and has previously had a good response to citalopram. She has developed recurrent depressive symptoms and one week ago was started on citalopram, $20 \mathrm{mg}$. On review her symptoms have not improved. Which is the most appropriate action?

A. Increase the citalopram dose.

B. Change to an alternative antidepressant.

C. Consider referral for psychological therapy.

D. Stop citalopram.

E. Continue to review mood weekly. 
4. A 67-year-old man is admitted with a painful and pulseless foot with an overlying infected ulcer. He has long-standing non-insulin-dependent diabetes mellitus. After surgical review a below-knee amputation is recommended. Following this amputation he appears tearful and upset on review at ward rounds. He seems to enjoy family visits and expresses enthusiasm about transfer to a rehabilitation unit. What is the most appropriate course of action?

A. Organise psychiatric review.

B. Consider trial of antidepressant.

C. Consider referral for psychological therapy.

D. Continue to monitor mood after transfer to rehab.

E. Organise psychiatric transfer.
5. A 50-year-old woman is being seen in clinic with suspected thyroid disease. She presents with a history of low mood, feeling cold and weight gain. Blood testing reveals that she is biochemically as well as clinically hypothyroid. What is the most appropriate way to address her mood symptoms?

A. Thyroid replacement therapy.

B. Trial of antidepressant.

C. Referral for psychological therapy.

D. Psychiatric review.

E. Organise psychiatric transfer.

This paper was originally published as part of the Psychiatry module in the RCPE Online Continuing Medical Education Programme. Online CME, including the anwers to these questions and further material, is available to Fellows and Members at: https:/lmembers.rcpe.ac.uk/cme.php

\section{UK CONSENSUS CONFERENCE ON DIABETES}

\section{I3 \& I4 May 2010 at the Royal College of Physicians of Edinburgh}

This two-day UK Consensus Conference, organised by the RCPE using evidence-based background papers and oral presentations, will produce a final consensus statement to influence diabetes care. Interactive discussions will help shape the final statement.

A multidisciplinary panel, chaired by Professor Roland Jung, Honorary Professor in Medicine at the University of Dundee and Visiting Professor at the University of Southampton, will address the following key questions:

- Who can prevent diabetes?

- What are the practical implications of developments in genetics?

- Which psychological interventions work?

- What after metformin - who cares?

- What are the best models of care for children and adolescents?

Abstracts based on the key questions are invited for poster presentation and should be submitted by Monday I March 2010.

In association with:

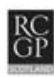
Romal College of
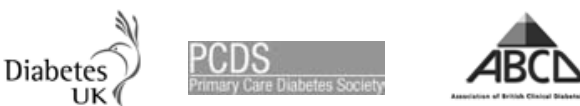

Full details on conference methodology, registration and abstract submission can be obtained from: http://events.rcpe.ac.uk

Or by contacting:

Margaret Farquhar, Consensus

Conference Co-ordinator, Education, Training and Standards Department, Royal College of Physicians of Edinburgh, 9 Queen Street, Edinburgh EH2 IJQ

Tel: + 44 (0) I3I 2473636

Email:m.farquhar@rcpe.ac.uk

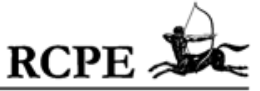

